\title{
Neuropathology of Temporal Lobe Epilepsy
}

\author{
Fahd Al Sufiani' ${ }^{1}$ and Lee Cyn Ang ${ }^{2}$ \\ ${ }^{1}$ Department of Pathology, London Health Sciences Centre, University of Western Ontario, London, ON, Canada N6A 5A5 \\ ${ }^{2}$ Department of Pathology (Neuropathology), London Health Sciences Centre, University of Western Ontario, London, \\ ON, Canada N6A 5A5 \\ Correspondence should be addressed to Lee Cyn Ang, leecyn.ang@gmail.com
}

Received 1 September 2011; Revised 20 January 2012; Accepted 7 February 2012

Academic Editor: Seyed M. Mirsattari

Copyright ( $) 2012$ F. Al Sufiani and L. C. Ang. This is an open access article distributed under the Creative Commons Attribution License, which permits unrestricted use, distribution, and reproduction in any medium, provided the original work is properly cited.

Pathologic findings in surgical resections from patients with temporal lobe epilepsy include a wide range of diagnostic possibilities that can be categorized into different groups on the basis of etiology. This paper outlines the various pathologic entities described in temporal lobe epilepsy, including some newly recognized epilepsy-associated tumors, and briefly touch on the recent classification of focal cortical dysplasia. This classification takes into account coexistent pathologic lesions in focal cortical dysplasia.

\section{Introduction}

Temporal lobe epilepsy (TLE) is the most common form of focal epilepsy [1]. In medical centers where epilepsy surgery is performed, neuropathologists often encounter temporal lobe resection specimens. Most patients scheduled for surgical resection present with intractable focal epilepsy and are selected for surgery based on clinical, electrophysiology, and neuroimaging criteria. The pathologic findings in these resected specimens represent different groups of conditions such as hippocampal sclerosis (HS), malformative lesions, tumors, ischemic lesions, old traumatic injuries, and inflammatory lesions. The objective of this paper is to outline the pathologic entities associated with TLE, while highlighting the recent classification of focal cortical dysplasia (FCD) and some recently described neoplastic entities.

\section{Hippocampal Sclerosis, Temporal Lobe Sclerosis, and Amygdaloid Sclerosis}

Hippocampal sclerosis, also known as Ammon horn sclerosis, is a common pathologic finding in surgical specimens from patients with TLE [2]. Although often used interchangeably with HS, the term "mesial temporal sclerosis" (MTS) is more appropriate for cases in which significant pathologic changes involve not only the hippocampus but also the amygdala and entorhinal cortex. The incidence of HS is variable in different studies, ranging from $48 \%$ to $73 \%$ [2-4]. Whereas the pathogenesis of HS is unknown, its occurrence after prolonged febrile seizures in early life has been implicated [5]. According to the International League Against Epilepsy (ILAE) Commission Report, HS is defined as neuronal loss and gliosis in hippocampal area CA1 (Sommer sector) and area CA4 (endplate/hilus/end folium) [6]. Histologically, the segmental loss of pyramidal neurons in area CA1 is severe, with less prominent neuronal loss in areas CA3 and CA4 (Figures 1(a) and 1(b)). The term "end folium sclerosis" is reserved for cases with neuronal loss and gliosis restricted mainly to area CA4. In approximately $50 \%$ of HS cases, granule cell dispersion in the dentate gyrus is demonstrated [6]. In addition to routine stains, such as hematoxylin-eosin (HE) and luxol fast blue/HE, immunohistochemistry for neuronal nuclei (NeuN) has proven valuable in delineating neuronal loss in area CA1 (Figure 1(a)) [7]. Immunohistochemistry for glial fibrillary acidic protein (GFAP) frequently highlights gliosis associated with neuronal loss (Figure 1(b)). Glial proliferation, particularly astrocytic, is believed to play a role in the glutamate excess linked to seizure generation in TLE $[3,8]$. Various semiquantitative methods have been devised to classify HS and MTS based on pathology findings $[9,10]$. Reviewing 178 cases of mesial temporal lobe epilepsy, Blümcke and associates [10] 


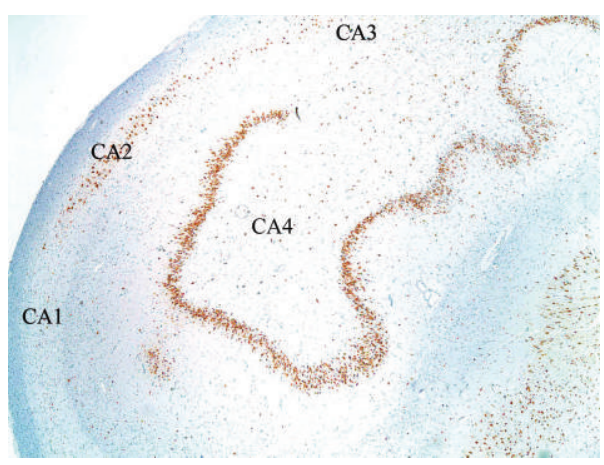

(a)

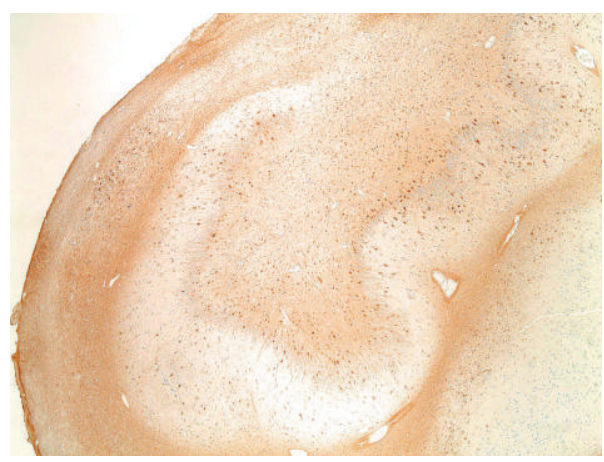

(b)

Figure 1: Hippocampal sclerosis. Neuronal loss in areas CA1, CA3, and CA4, with gliosis, (a) NeuN immunoreactivity. (b) glial fibrillary acidic protein immunoreactivity. Original magnification $\times 20$.

suggested that the patterns of MTS be categorized into (1) no MTS, (2) MTS type 1 (neuronal loss predominantly in areas CA1 and CA4), (3) MTS type 2 (CA1 sclerosis), and (4) MTS type 3 (end folium sclerosis). Cases categorized as MTS types 2 and 3 were noted to have worse postoperative seizure outcome (Engel classification) compared to MTS type 1 [10].

Thom and associates [11] used the term "temporal lobe sclerosis" to describe HS cases with definite neuronal loss and laminar gliosis in layers II/III of the temporal neocortex. Temporal lobe sclerosis has little influence on postsurgical seizure outcome and, therefore, has been regarded as an extension of HS rather than a separate entity [11]. Although a milder degree of neuronal loss in the amygdala is often observed in HS [12], "amygdaloid" or "amygdalar" sclerosis applies to severe neuronal loss with gliosis in the amygdala (especially in the lateral nucleus). Cases of amygdaloid sclerosis have also been identified without HS [13]. Such cases are believed to form a distinct group, with no clinical history of early brain insult such as febrile convulsion [13]. Given that interpretation of the degree of neuronal loss in the amygdala is often subjective, the incidence of amygdaloid sclerosis reported is highly variable, ranging from $6 \%$ to $100 \%$ [13$15]$.

The coexistence of an additional extrahippocampal brain pathology (excluding temporal lobe sclerosis and amygdaloid sclerosis) in HS cases, referred to as "dual pathology," has a reported prevalence of $5 \%$ to $34 \%$ [2, 16-19]. In a study of TLE by Tassi and associates [2], only 34 cases (29\%) of 117 HS cases were without dual pathology [2]. The rest were cases of dual pathology with additional lesions such as FCD and tumors. There are rare cases in the literature of HS coexisting with intrahippocampal pathology such as FCD involving only the end folium [20].

\section{Malformations of Cortical Development and Focal Cortical Dysplasia}

The term "malformations of cortical development" (MCD) encompasses developmental abnormalities of the cortex representing the broad spectrum of clinicopathologic changes attributable to various pathogenetic mechanisms during prenatal and postnatal development [21]. These mechanisms can influence different developmental processes such as cell proliferation/apoptosis, neuronal migration, and cortical organization. Although numerous malformations have been attributed to genetic mutations or inborn metabolic errors (e.g., peroxisomal or mitochondrial disorders), other acquired causal factors affecting embryonic and fetal development have to be considered [22]. Focal cortical dysplasia is a subtype of MCD that is cited as a common cause of medically intractable, chronic epilepsy in children and young adults [21]. It includes a wide range of lesions, including cortical dyslamination, cytoarchitectural abnormalities, and underlying white matter disturbance. In our study of focal epilepsy patients with pathological diagnosis of FCD, the electrophysiology data, medical imaging features, and neuropsychology data identified the temporal lobe as the origin of epileptogenesis in 50\% of the cases [23]. The prevalence of FCD reported in various studies of focal epilepsy involving TLE ranges from $9 \%$ to $45 \%[2,17,24-26]$. Focal cortical dysplasia can be present alone or in association with other pathologic entities such as glioneuronal tumors or HS [27]. Other examples of MCD include agyria, pachygyria, porencephaly, grey matter heterotopia, and polymicrogyria, which can be multifocal or more diffuse $[10,28]$ (Figures 2(a), 2(b), and $2(\mathrm{c}))$.

Similar to HS, a history of febrile seizure has been statistically linked to patients with MCD, particularly FCD [2]. Some authors have proposed the following explanations for the association between febrile seizure, MCD, and HS: (a) MCDs have a predisposition for prolonged childhood febrile seizure, which can lead to HS, (b) MCDs are responsible for repeated seizures that cause secondary hippocampal damage, and (c) MCDs and HS stem from common embryonic damage [2, 17, 29]. The findings of Tassi and associates [2] support the notion that MCD can predispose to febrile seizure and that repeated seizures result in HS.

Resected FCD specimens may be normal on macroscopic observation. However, in some cases, the cortex appears thickened, with poor demarcation from the underlying white matter. Careful gross examination, with generous sampling and correlation with clinical (including electroencephalography and operative findings) and neuroimaging data, is 


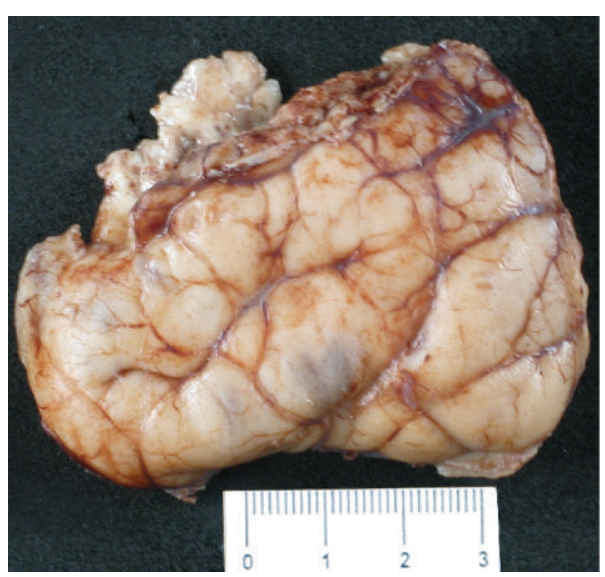

(a)

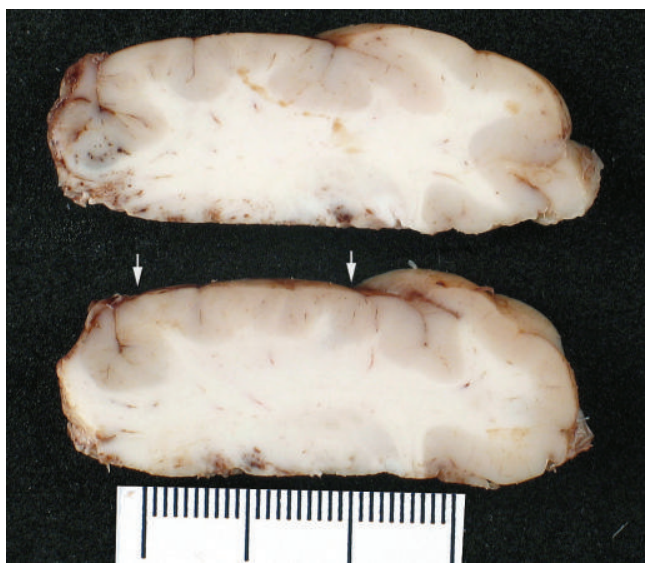

(b)

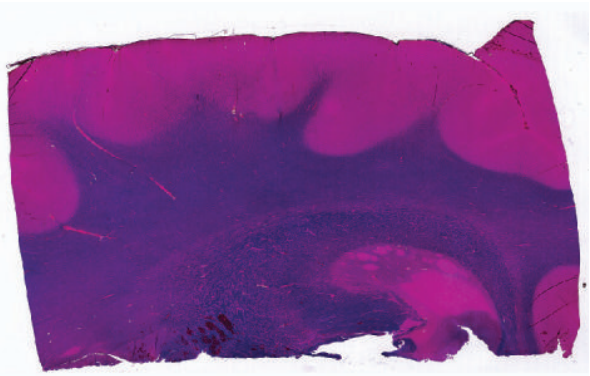

(c)

FIGURE 2: A cortical resection specimen from a child with hemimegalencephaly shows polymicrogyria. (a) external surface. (b) coronal sections, excessive convolution of cortical ribbon with shallow fused sulci (area between arrows). (c) micrograph (luxol fast blue-hematoxylin and eosin stain), several small wisps of subcortical white matter extending up to the cortical ribbon.

considered a reasonable standard of practice. Recently, a standardized protocol has been developed for the pathologic workup of FCD [30]. Samples are snap-frozen for molecular biology or genetic analysis when necessary, and the remaining (majority) resected specimen is fixed in 10\% formalin. The specimen is then cut into thin slices oriented perpendicular to the cortical surface. Slices are carefully examined, photographed, and sampled before embedding in paraffin. The 4 to $7 \mu \mathrm{m}$-thick paraffin sections are routinely stained with hematoxylin-eosin. Additional staining or immunohistochemistry is performed on selected sections to assess cortical architecture (cresyl violet, NeuN), myelination (luxol fast blue), and cytologic features (microtubule-associated protein 2 [MAP2], phosphorylated/nonphosphorylated neurofilament protein).

Focal cortical dysplasia is categorized into FCD type I (cortical dyslamination) and FCD type II (with the addition of dysmorphic neurons and/or balloon cells) according to the Palmini classification [31]. An analysis of inter- and intraobserver agreement using the Palmini classification found that the overall agreement was moderate and less reproducible for FCD type I, even among experienced neuropathologists [32]. The greatest concordance was observed for FCD type II, in which dysmorphic neurons and/or balloon cells were identified. There are often difficulties in drawing a clear distinction between normal and dyslaminated cortex, owing to the cytoarchitectural heterogeneity of different subregions of the temporal lobe (e.g., Brodmann areas 21, 38, 28, $27,35,20,30$, and 37), thus making differentiation between normal cytoarchitectural variation and mild cortical disorganization quite subjective. For example, according to Ding and associates, the granular layer of the temporal cortex is quite variable in the different Brodmann areas, and it diminishes towards the temporopolar area (Brodmann 38) [33]. In most studies on TLE, however, the Brodmann areas have not been specified in the surgical resections and the subsequent histological examination. The ILAE Task Force has recently introduced a consensus classification system based on correlations between neuropathology findings, imaging data, electrophysiology features, and postsurgical seizure control [34]. The ILAE classification highlights a group of FCDs with coexistent pathology (FCD type III) that demonstrates favorable postsurgical outcomes. This classification divides FCD into three clinicopathologic types (see Table 1) [35]. In FCD type $\mathrm{I}$, there is isolated neocortical dyslamination (Figure 3(a)) that may be either radial (type Ia), tangential (type Ib), or a combination of both (type Ic). In FCD type II (Figure 3(b)), there is isolated neocortical dyslamination, but in addition, dysmorphic neurons (type IIa) or balloon cells, with or without dysmorphic neurons (type IIb), are identified. Whereas FCD types I and II are largely alluded to in the Palmini classification, the ILAE Task Force introduced 
TABLE 1: International league against epilepsy consensus classification system for focal cortical dysplasia (FCD) [34].

\begin{tabular}{|c|c|c|c|c|}
\hline FCD type I: isolated & $\begin{array}{l}\text { FCD Ia: abnormal radial } \\
\text { cortical lamination }\end{array}$ & \multicolumn{2}{|c|}{ FCD Ib: abnormal tangential cortical lamination } & $\begin{array}{l}\text { FCD Ic: abnormal radial } \\
\text { and tangential cortical } \\
\text { lamination }\end{array}$ \\
\hline FCD type II: isolated & \multicolumn{2}{|c|}{ FCD IIa: dysmorphic neurons } & \multicolumn{2}{|c|}{ FCD IIb: dysmorphic neurons and balloon cells } \\
\hline $\begin{array}{l}\text { FCD type III: } \\
\text { associated with } \\
\text { principal lesion }\end{array}$ & $\begin{array}{l}\text { FCD IIIa: cortical lamination } \\
\text { abnormalities in the temporal } \\
\text { lobe associated with } \\
\text { hippocampal sclerosis }\end{array}$ & $\begin{array}{l}\text { FCD IIIb: cortical lamination } \\
\text { abnormalities adjacent to a } \\
\text { glial or glioneuronal tumor }\end{array}$ & $\begin{array}{l}\text { FCD IIIc: cortical } \\
\text { lamination abnormalities } \\
\text { adjacent to vascular } \\
\text { malformation }\end{array}$ & $\begin{array}{l}\text { FCD IIId: cortical } \\
\text { lamination } \\
\text { abnormalities adjacent } \\
\text { to any other lesion } \\
\text { acquired during early life } \\
\text { (e.g., trauma, ischemic } \\
\text { injury, and encephalitis) }\end{array}$ \\
\hline
\end{tabular}

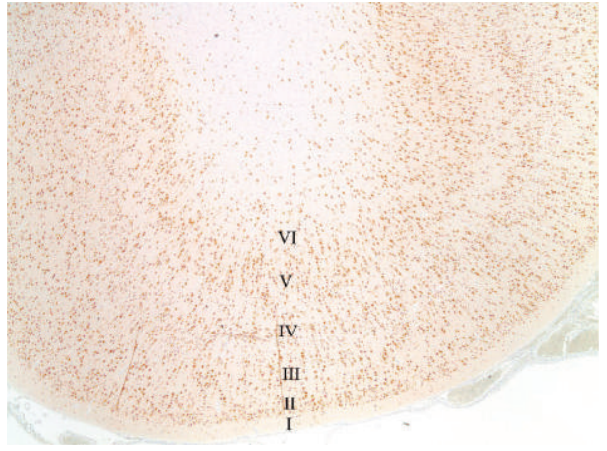

(a) FCD type Ia

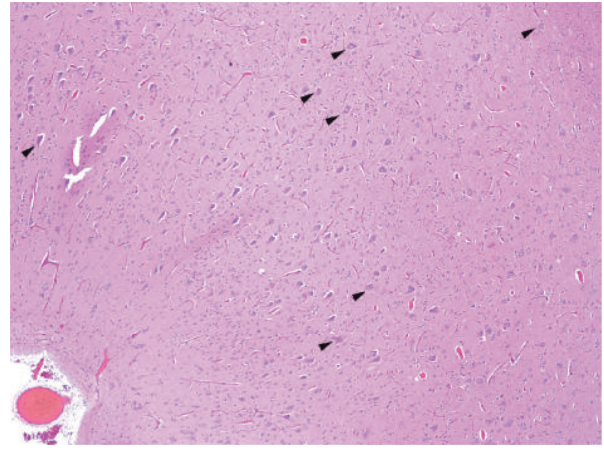

(b) FCD type IIa

FIGURE 3: Focal cortical dysplasia (FCD). (a) FCD type Ia shows microcolumnar arrangements of cortical neurons with a preservation of cortical layering. NeuN immunoreactivity. Original magnification $\times 20$. (b) FCD type IIa, cortical laminar disorganization and dysmorphic neurons (arrowhead), distributed throughout the entire cortical thickness (sparing the molecular layer) and subjacent white matter. Hematoxylin-eosin stain. Original magnification $\times 40$.

the classification of FCD type III, which takes into account the presence of other epileptogenic lesions in association with FCD type I, such as HS (type IIIa), epileptogenic tumors (type IIIb), vascular malformation (type IIIc), or lesions acquired during early life, for example, as a result of trauma, ischemic injury, or encephalitis (type IIId) [34].

The ILAE Task Force consensus classification also provides standardized descriptions for abnormal cell types such as dysmorphic neurons, balloon cells, and hypertrophic neurons, among others [34]. Histologically, dysmorphic neurons show a large soma and nucleus, with abnormally aggregated Nissl substance displaced toward the cell membrane and cytoplasmic accumulation of phosphorylated and nonphosphorylated neurofilament protein (Figures 4(a) and 4(b)). Balloon cells show a large soma with an eccentric nucleus and abundant, eosinophilic, glassy cytoplasm on HE staining (Figure 5). These cells are considered to be indeterminate or transitional cell types because they often express both neuronal and glial markers [36]. In addition, descriptive and diagnostic terms, such as dysplasia, heterotopia, hamartoma, ectopia, dyslamination, dual pathology, double pathology, and principal lesions have been further defined to ensure standardized pathologic reporting of FCD.

From the cases of our previous FCD study [23], we selected 57 surgical resections from the temporal lobe in which microscopic slides were available for pathology review. The histologic subtypes for these FCD cases were assigned based on the Palmini classification and then reassigned according to the ILAE classification. Isolated FCD was observed in $70 \%$ of the cases, and the rest were associated with other lesions. With one exception, lesions in all cases with other associated lesions were localized to the temporal lobe. Using the Palmini classification, type IIB was the most common subtype (21 cases, $37 \%$ ). There were 16 cases (28\%) associated with other pathologic lesions such as hippocampal sclerosis, low-grade neuroepithelial tumors, hamartomas, and destructive lesions. With the ILAE classification, FCD type IIB remained the most common subtype, but seven cases of FCD type I were reclassified as type III because of the presence of another principal lesion. There were nine cases (16\%) of FCD type II with coexistence of other principal lesions. Three FCD type II cases (dual pathology) were associated with hippocampal sclerosis, and the other six cases (double pathology) were associated with either low-grade glioneuronal tumor, hamartomas, or destructive lesions. The major difference with the ILAE classification is that it recognizes the coexistence of other principal lesions in FCD, which were present in $28 \%$ of our cases [37].

Microdysgenesis constitutes a form of microscopic malformation in cortical development characterized mainly by 


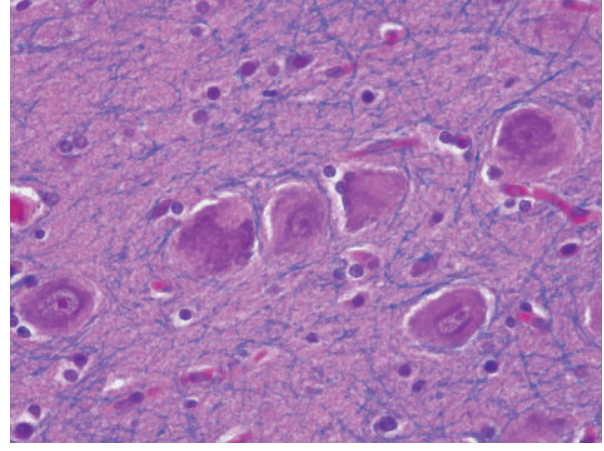

(a)

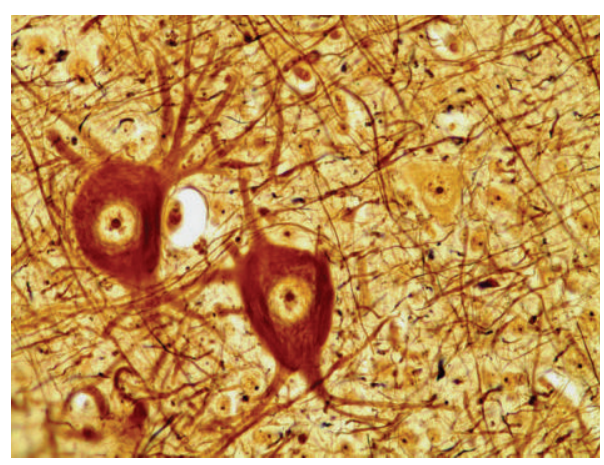

(b)

Figure 4: Dysmorphic neurons. (a) hematoxylin-eosin stain. Original magnification $\times 400$. (b) Bielschowsky stain. Original magnification $\times 400$.

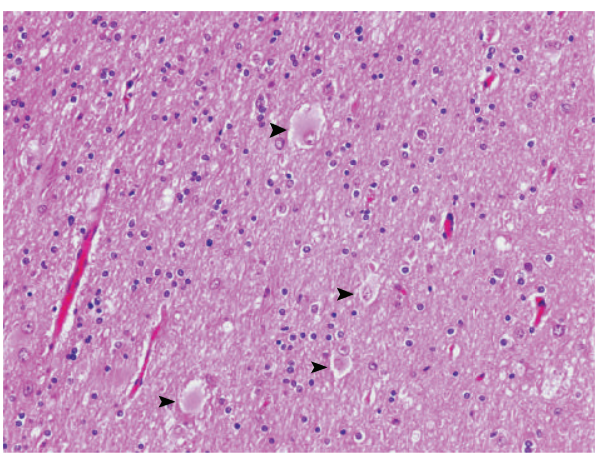

FIGURE 5: Balloon cells. Hematoxylin-eosin stain. Original magnification $\times 200$.

mild cortical cytoarchitectural abnormalities and an increase in heterotopic neurons in both cortical layer I and the subcortical white matter $[38,39]$. Of all of the histologic features in microdysgenesis, the increase in heterotopic neurons in subcortical white matter appears to be the most consistent finding [39]. The actual quantitative data for subcortical neuronal counts remain highly variable between studies, depending on the different cortical regions evaluated, and the processing techniques and morphometric methods applied [40, 41]. However, Kasper and associates [42] reported that a count of more than 10 white matter neurons per highpower field applied to temporal lobe sections can be useful in differentiating TLE cases from normal autopsy control cases [42]. In the ILAE classification, the term microdysgenesis has been omitted, owing to the lack of a precise definition, and instead "mild forms of cortical malformations" are used to define an excess of neurons in the white matter [43].

\section{Meningioangiomatosis, Vascular Malformation, and Hamartomas}

Meningioangiomatosis (MA) is a hamartomatous lesion that can occur sporadically or in association with neurofibromatosis type 2. Sporadic MA commonly presents as a plaque-like mass in the setting of chronic epilepsy [44].

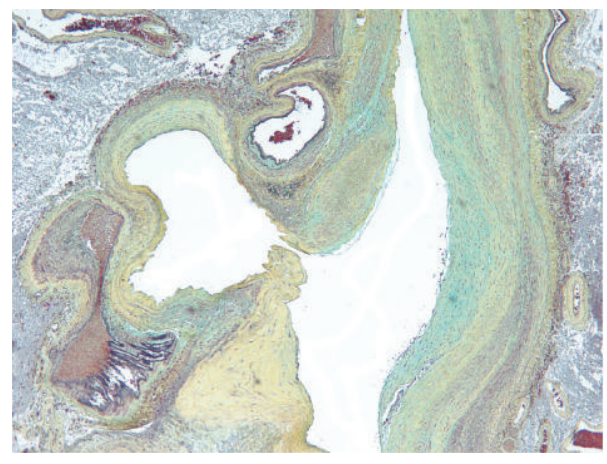

FIGURE 6: Arteriovenous malformation comprises of mixture of arteries, veins, and abnormal vessels of variable wall thickness and caliber (arterialized veins) with intervening gliotic brain tissue. Extensive perilesional gliosis, calcification, and microhemorrhages, with hemosiderin deposition (not shown) are common features in vascular malformation. Movat stain. Original magnification $\times 100$.

Interestingly, MA associated with neurofibromatosis type 2 is usually asymptomatic and rarely associated with seizures. On microscopy, MA shows the proliferation of meningothelial and fibroblastic cells arranged around cortical blood vessels, with occasional extension into the white matter [45]. Psammomatous calcification is frequent, and the intervening cortical tissue often shows gliosis. When the vascular component predominates, it mimics a vascular malformation. One report documented an overlying meningioma in $42 \%$ of MA cases, and in this setting, the authors considered the MA component to be neoplastic in nature [46]. Surgical excision of the lesion, including the adjacent cortex, is regarded as curative, even though seizures can persist in a number of patients [44].

In surgical series of focal epilepsy, vascular malformation, consisting mostly of arteriovenous malformations (Figure 6) and cavernous hemangioma, is less common than expected. However, studies on vascular malformation report that approximately $25 \%$ of patients present with epilepsy [47]. Cavernous hemangioma in the temporal lobe can be treated by extended lesionectomy, with excellent postoperative seizure control, and in cases with concurrent hippocampal 


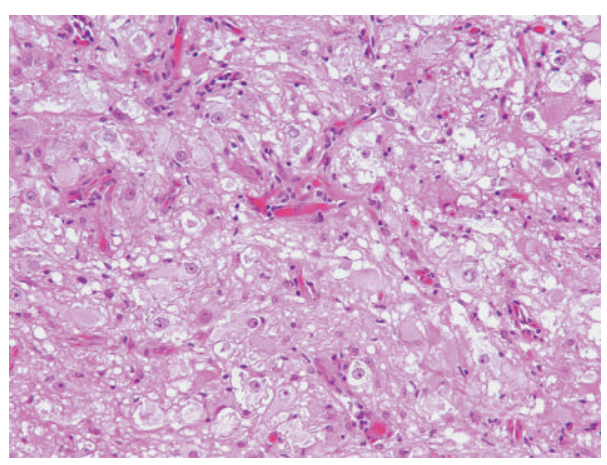

(a)

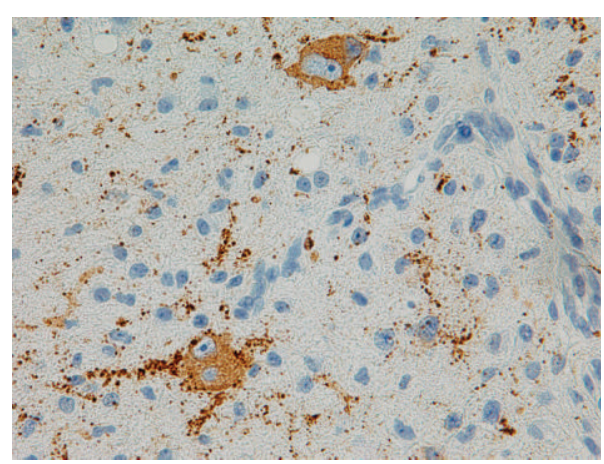

(b)

Figure 7: Ganglioglioma. (a) mixed population of neoplastic glial and ganglion (neuronal) cells. Hematoxylin-eosin stain. Original magnification $\times 200$. (b) examples of neoplastic, binucleated neurons immunoreactive for synaptophysin. Original magnification $\times 400$.

sclerosis (dual pathology), the resection should also include the mesial temporal structures [48].

Hamartoma is a nonneoplastic, nodular proliferation of disorganized mature tissues that are native to the site of origin. In the central nervous system, this usually refers to glioneuronal hamartomas, which have rarely been described in TLE [49]. The lesion is usually well circumscribed, with a bland, nonproliferative glial component, and a neuronal component showing minimal cellular atypia. Occasionally, it is difficult to distinguish the less cellular variants of ganglioglioma (GG) or dysembryoplastic neuroepithelial tumor (DNT) from hamartomas, suggesting that some of these entities may fall within the same histogenetic spectrum [50]. Focal cortical dysplasia has been identified in the adjacent cortex in some hamartomas [49]. Interestingly, rare cases of hypothalamic hamartoma have been reported to manifest with a syndrome similar to TLE [51].

\section{Tumors}

In general, tumor-induced epilepsy of the temporal lobe tends to present earlier in life (i.e., childhood and young adulthood). The prevalence of tumors reported in epileptic patients is variable, and may be as high as $30 \%[2,52]$. Whereas any slow-growing tumor (e.g., meningioma and glioma) can be associated with focal epilepsy, glioneuronal tumors have been identified as the most common epilepsyinducing tumor [52]. However, in one study of pediatric tumor-related TLE, pilocytic astrocytoma was identified as most common tumor (41\%), followed by GG (25\%) [53]. Given that TLE-associated tumors are frequently of low grade, general and seizure outcomes after surgical resection are good for most patients.

Occasionally, two tumor types can form a composite tumor such as in the case of DNT and GG, or GG with pleomorphic xanthoastrocytoma (PXA) [54, 55]. A recent study revealed that the most common pathology coexisting with tumor-related epilepsy is FCD, followed by hamartia and hippocampal sclerosis [56]. Ganglioglioma and DNT are the tumors most frequently associated with $\operatorname{FCD}[2,52]$.
The glioneuronal tumors associated with TLE include GG, gangliocytoma, DNT, and the novel entity of papillary glioneuronal tumor.

Ganglioglioma (World Health Organization (WHO) grade I) is the most common tumor identified in chronic epilepsy and is frequently located in the temporal lobe, although it may arise elsewhere in the central nervous system $[52,56]$. This is a slow-growing tumor, mostly affecting children and young adults. Gangliogliomas may appear solid, cystic, or a combination of the two. Calcification may be observed macroscopically but is more likely to be visible only at the microscopic level. Histologically, this tumor is composed of neoplastic glial and neuronal (ganglion) cells [57]. Glial and neuronal components may be evenly admixed throughout the tumor or may be unevenly distributed, resulting in significant tumor heterogeneity (Figure 7(a)). Neoplastic ganglion cells in the neuronal component are usually large, often bi- or multi-nucleated, and dysplastic in appearance, with an abnormal distribution of Nissl substance. The glial component of this tumor is quite variable and usually forms the proliferative fraction of the tumor. Morphologically, the glial component can resemble fibrillary astrocytoma, oligodendroglioma, or pilocytic astrocytoma. Perivascular lymphocytosis, focal calcification, and eosinophilic granular bodies are common findings. Mitoses are rare, and necrosis is predominantly absent. Although neuronal markers such as MAP2, NeuN, neurofilaments, and synaptophysin are useful for identification of the neuronal component, these markers cannot differentiate neoplastic neurons from native, nonneoplastic ones (Figure 7(b)). Immunohistochemistry for CD34 could be helpful in distinguishing neoplastic ganglion cells from entrapped, nonneoplastic neurons [58]. Ganglioglioma is usually associated with a good prognosis, but occasional anaplastic GG with malignant transformation of the glial component has been reported (e.g., $5 \%$ in a study by Blümcke and Wiestler) [59, 60].

Dysembryoplastic neuroepithelial tumor (WHO grade I) is a supratentorial, heterogeneous, and glioneuronal neoplasm in children and young adults, with a predilection for the temporal lobe and often associated with drug-resistant 


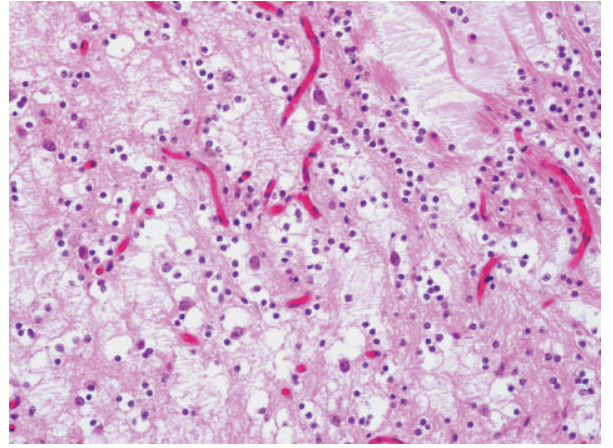

FIGURE 8: Dysembryoplastic neuroepithelial tumor. Oligodendroglia-like cells arranged in columns with occasional mature neurons and myxoid background. Hematoxylin-eosin stain. Original magnification $\times 200$.

partial seizures [61]. It is predominantly cortical and typically exhibits a complex columnar and multinodular architecture [62]. Grossly, there is expansion of the cortex, with isolated or multiple nodules and frequent microcystic changes. Histologically, there is a monomorphous population of round nuclei with scant cytoplasm (oligodendroglialike cells) arranged in columns oriented perpendicular to the cortical surface. Occasional "floating" mature neurons are seen within a myxoid extracellular matrix between columns of oligodendroglia-like cells (Figure 8). These oligodendroglia-like cells express neuronal markers (e.g., synaptophysin). The complex variant of DNT displays heterogeneous astrocytoma, oligodendroglioma, and neuronal components [63]. Immunochemistry for CD34, nestin, and calbindin is often helpful in differentiating DNT from other gliomas, especially in small specimens [61]. The adjacent cortical tissue frequently shows cortical dysplasia [64]. Dysembryoplastic neuroepithelial tumor is a low-grade tumor with good outcome in terms of seizure-free interval and recurrence after complete surgical excision [61]. However, rare malignant transformation has been reported $[65,66]$.

The recently described papillary glioneuronal tumor (WHO grade I) often presents with seizures. It is reported to be rare, but we identified one case in our review of 57 FCD cases [37]. On microscopy, it consists of pseudopapillae structures with a hyalinized, vascular core enclosed by flat/ cuboidal GFAP-positive glial cells (Figures 9(a) and 9(b)). The interpapillary areas show sheets of round neurocytic cells which are positive for neuronal markers (e.g., synaptophysin, NeuN) (Figure 9(c)) [67]. A favorable prognosis is expected after gross total removal [68].

A number of slow-growing, low-grade tumors, such as pilocytic astrocytoma, diffuse astrocytoma, oligodendroglioma, and ependymoma, are known to present with focal epilepsy. In this paper, however, we will discuss only three of these tumor types specifically. An often encountered tumor in children and young adults with protracted history of seizures, PXA (WHO grade II) is regarded as an astrocytic neoplasm with limited neuronal differentiation. This tumor is found mostly in the supratentorial compartment, particularly in the temporal lobe. It is typically superficial and often involves the adjacent meninges, with occasional dural invasion. The tumor has both solid and cystic components, and on neuroimaging can appear as a "cyst with mural nodule." The most characteristic histologic feature is the presence of large, pleomorphic, and lipidized cells (Figure 10(a)), which are often individually surrounded by a reticulin network [69]. Perivascular lymphocytosis, eosinophilic granular bodies, and Rosenthal fibers can be seen. Pleomorphic xanthoastrocytoma tumor cells are GFAP-positive, with variable immunoreactivity for neuronal markers [70] (Figure 10(b)). The prognosis for this tumor in young individuals is generally favorable [71]. However, there are rare examples of anaplastic PXA with increased mitotic activity and tumor necrosis [72-74]. Some histologic features of anaplastic PXA, including the presence of large pleomorphic cells and reticulin deposition, make differentiation from giant cell glioblastoma a diagnostic challenge [75].

Angiocentric glioma ( WHO grade I), another distinctive tumor included in the recent WHO classification for central nervous system tumors, has been reported in children and young adults presenting with chronic, intractable, partial seizures [76]. This is a slow-growing, superficial, cerebral tumor that can be found in the temporal lobe (we identified one case in our review of 57 TLE cases) [37]. Angiocentric glioma is characterized histologically by an angiocentric growth pattern of monomorphous bipolar cells showing immunoreactivity for GFAP, S-100 protein, and vimentin (Figures 11(a) and 11(b)). Some of these tumors show immunoreactivity for epithelial membrane antigen and the presence of intracellular microlumens with microvilli and cilia ultrastructurally, findings which support ependymal differentiation [77] (Figure 11(c)).

While isomorphic astrocytoma is considered a variant of diffuse astrocytoma (WHO grade II), its clinical behavior is more akin to that of a WHO grade I astrocytoma $[78,79]$. This tumor is mainly described in patients with chronic epilepsy who experience long survival and low recurrence rate. Histologically, this tumor shows an isomorphic, sparse population of small glial cells with rounded nuclei. Whereas tumor cells appear infiltrative, there is an absence of nuclear atypia, mitotic activity, and necrosis. This tumor shows immunoreactivity for GFAP but not for MAP2 or CD34.

\section{Posttraumatic, Destructive, and Ischemic Lesions}

Approximately $20 \%$ of symptomatic epilepsy is attributed to some kind of trauma [80]. Risk factors for posttraumatic seizures include alcoholism, age, penetrating dural injury, intracranial hemorrhage, depressed skull fracture, and focal neurologic deficits. Seizures may present beyond the first week after injury, mostly within 18 months after injury [81]. Late posttraumatic seizures are thought to result from cortical damage caused by free radicals generated by iron deposition from extravasated blood, along with increased excitotoxicity owing to glutamate accumulation [82]. In most surgical resection specimens of posttraumatic epilepsy, the tissue consists of cystic lesions that involve the crests of gyri 


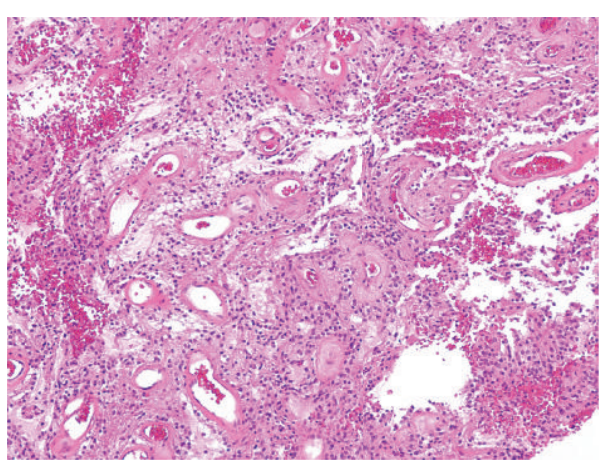

(a)

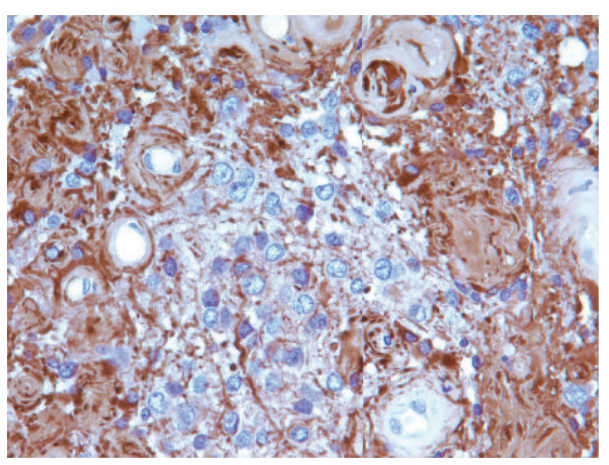

(b)

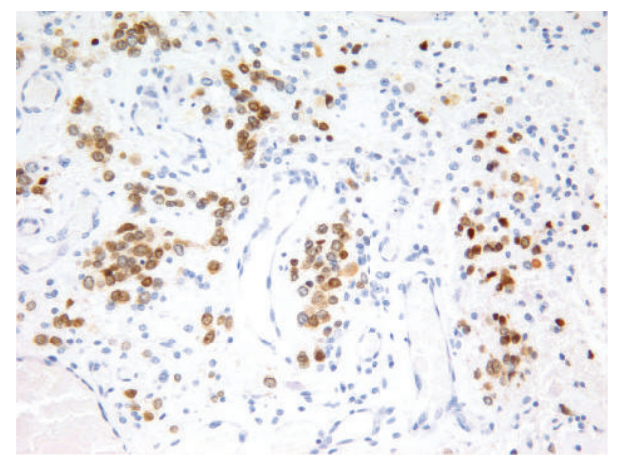

(c)

FIGURE 9: (a) pseudopapillae structures composed of hyalinized blood vessels and surrounded by glioneuronal cells. Papillary glioneuronal tumor may mimic vascular malformation. Hematoxylin-eosin stain. Original magnification $\times 100$. (b) the glial component around blood vessels. Glial fibrillary acidic protein (GFAP). Original magnification $\times 400$. (c) neuronal component in the interpapillary area. NeuN immunostain. Original magnification $\times 200$.

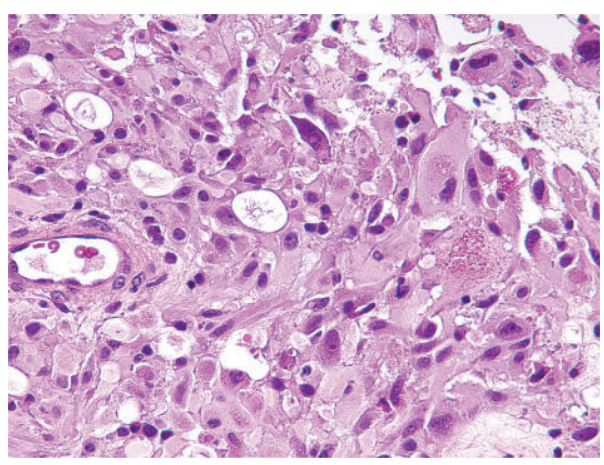

(a)

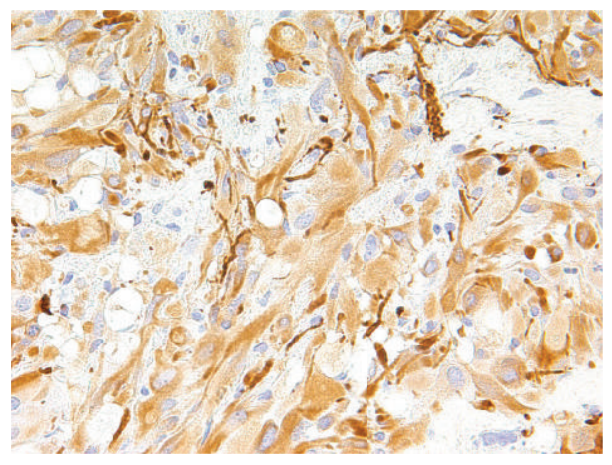

(b)

FIGURE 10: (a) pleomorphic xanthoastrocytoma with large, pleomorphic, and lipidized glial cells. Hematoxylin-eosin stain. (b) glial fibrillary acidic protein immunoreactivity (astrocyte marker). Original magnification $\times 600$.

with hemosiderin deposition (Figure 12). These lesions are often localized in the inferior frontal and temporal lobes, where cortical contusions are common.

The prevalence of epilepsy as a late sequela of adult stroke has been estimated at $3 \%$ to $10 \%$, depending on the definition of epilepsy [83]. The predominant seizure type is partial and can be the result of either cerebral infarct or hemorrhage [84]. The remote ischemic infarct is cystic with central cavitation surrounded by a zone of prominent gliosis.
Hemosiderin deposition may be evident in old infarcts with a hemorrhagic component. There is no clear association between the incidence of seizures and the location of the infarct. Chronic ischemic damage of the cortex may display prominent cortical atrophy, with a loss of neurons and gliosis without cavitation. Porencephalic cysts are usually the result of infarct in the fetal brain during gestation. This type of cyst is localized on one cerebral hemisphere, connecting one ventricle to the brain surface [85]. Quite often in both 


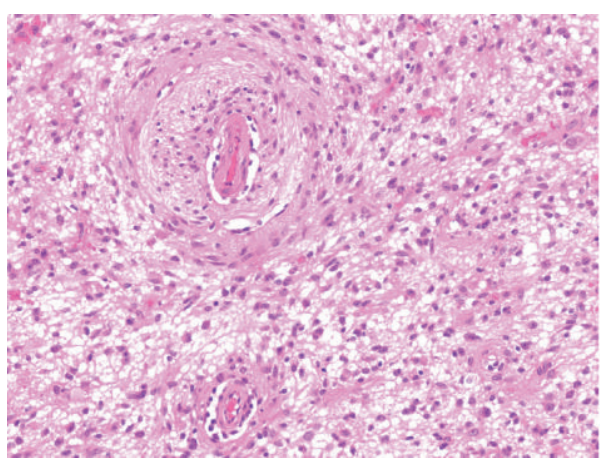

(a)

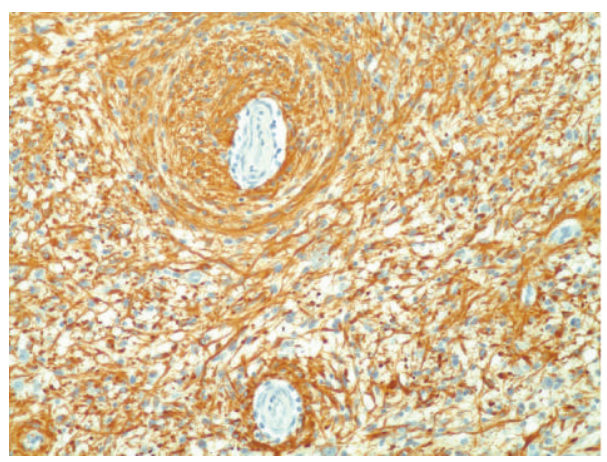

(b)

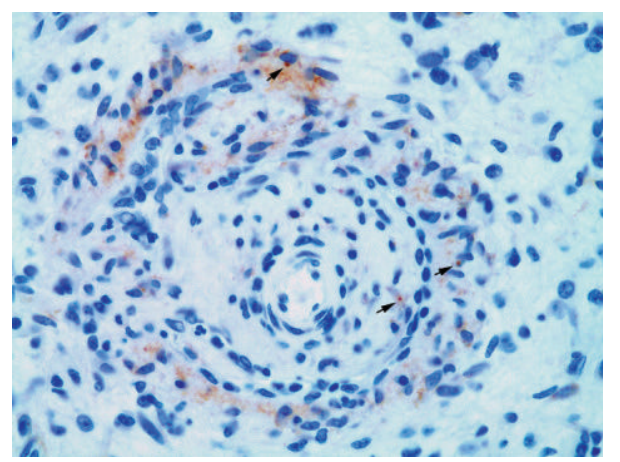

(c)

FIgURE 11: Angiocentric glioma. (a) angiocentric growth pattern of monomorphous bipolar cells. Hematoxylin-eosin stain. (b) corresponding section stained for glial fibrillary acidic protein shows cell processes wrapped around blood vessels. Original magnification $\times 200$. (c) epithelial membrane antigen shows a dot-like immunoreactivity (arrow). Original magnification $\times 400$.

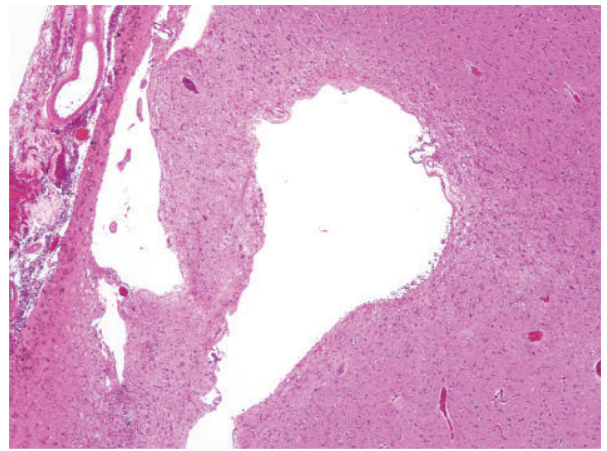

(a)

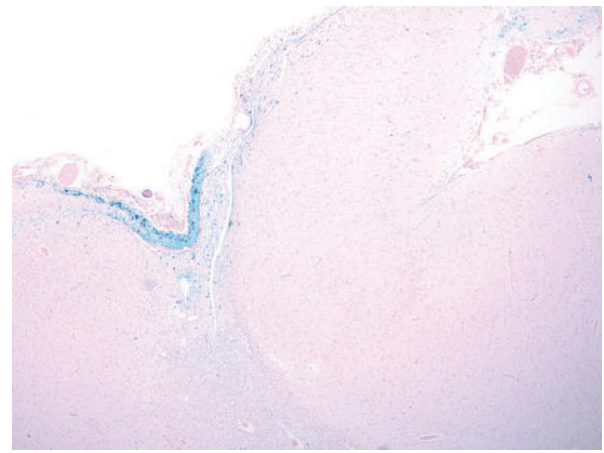

(b)

Figure 12: Old trauma. (a) cortical cystic lesion with hemosiderin pigments. Hematoxylin-eosin stain. Original magnification $\times 40$. (b) superficial iron deposit within the cortical tissue. Perl Prussian blue stain. Original magnification $\times 20$.

posttraumatic and postischemic injuries, the cystic and hemorrhagic components resolve, with only scar lesions remaining [25].

\section{Inflammatory Lesions}

Localized inflammatory lesions, such as brain abscesses, granulomas (tuberculosis), and parasitic cysts (neurocysticercosis and hydatid cysts) are important causes of focal epilepsy, particularly in developing countries [86, 87]
(Figure 13). Some cases of chronic encephalitis affecting the temporal lobe (limbic encephalitis) can also present with chronic seizures. Like other cases of chronic encephalitis, there are perivascular lymphocytic infiltrate and microglial nodules in the brain parenchyma (Figures 14(a) and 14(b)). The role of the inflammatory process and the immune response in epileptogenesis has been well documented in animal models and is considered to be a plausible cause of epileptogenesis in humans $[88,89]$. It should be noted that some degree of chronic leptomeningeal and perivascular 


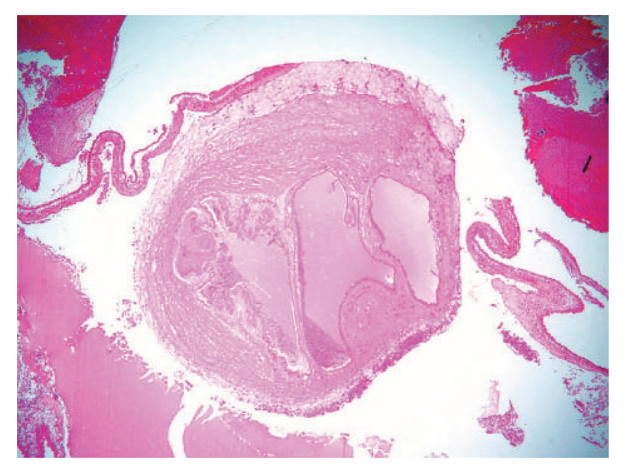

FIGURE 13: Neurocysticercosis characterized by the presence of undulating, laminated, and membranous wall of a cysticercus. Hematoxylin-eosin stain. Original magnification $\times 40$.

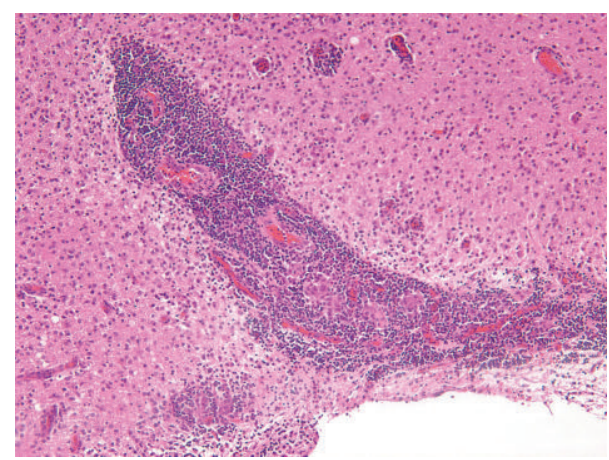

(a)

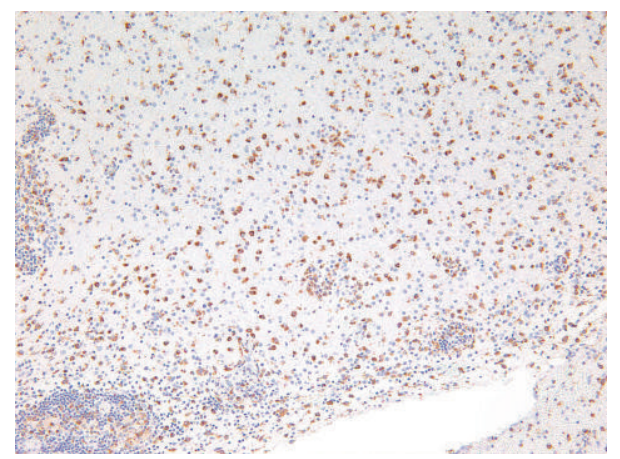

(b)

FIGURE 14: (a) perivascular lymphohistiocytic infiltrate in meningoencephalitis from a patient with temporal lobe epilepsy. Hematoxylin-eosin stain. (b) diffuse microglial infiltration with the formation of nodules. Immunostaining for CD68. Original magnification $\times 100$.

parenchymal inflammation may be secondary to invasive seizure monitoring and should not be mistaken for chronic encephalitis or meningitis.

Rasmussen encephalitis is a rare syndrome that exemplifies the association of chronic epilepsy and chronic encephalitis. The disease is characterized by the sudden onset of seizures (unilateral motor seizures or epilepsia partialis continua) in previously healthy individuals, typically within the first 2 decades of life $[90,91]$. As Rasmussen encephalitis is unihemispheric, the pathology is not restricted to the temporal lobe. There is usually extensive involvement of one hemisphere with active chronic inflammation in the earlier stages and scarring and atrophy in later stages [91]. Inflammatory cells in the cortex are mostly $\mathrm{T}$ lymphocytes with perivascular and perineuronal aggregates. There are also microglial nodules, which are pervasive in the earlier stages. In the later stages, reactive astrocytes are more predominant [92].

\section{Conclusion}

There is a wide range of pathologic findings in TLE, many of which are quite complex because more than one type of lesion is often identified in a single surgical resection specimen. The careful examination of these specimens, with the application of techniques such as immunohistochemistry, has provided improved classification of FCD and more accurate diagnosis of neoplastic and nonneoplastic conditions. This will enhance our understanding of the pathogenesis of TLE and ultimately lead to better management strategies. It is also pertinent for pathologists to understand that an accurate pathologic diagnosis cannot be attained without a correlation between pathologic and clinical findings, electrophysiologic data, and neuroimaging data. Finally, despite careful examination, there will remain a percentage of pathologic specimens that defy specific diagnosis $[2,25]$. In this group, we include cases of "bizarre" or "unusual" lesions, which remain unclassifiable based on our current understanding of neuropathology as well as cases with no obvious abnormalities, which may be explained by the collection of nonrepresentative resection specimens or by the diagnosis not being attainable with current methods.

\section{References}

[1] S. Wiebe, "Epidemiology of temporal lobe epilepsy," Canadian Journal of Neurological Sciences, vol. 27, no. 1, pp. S6-S10, 2000.

[2] L. Tassi, A. Meroni, F. Deleo et al., "Temporal lobe epilepsy: neuropathological and clinical correlations in 243 surgically treated patients," Epileptic Disorders, vol. 11, no. 4, pp. 281292, 2009.

[3] I. Blümcke, M. Thom, and O. D. Wiestler, “Ammon's horn sclerosis: a maldevelopmental disorder associated with temporal lobe epilepsy," Brain Pathology, vol. 12, no. 2, pp. 199-211, 2002.

[4] M. C. Lee, G. M. Kim, Y. J. Woo et al., "Pathogenic significance of neuronal migration disorders in temporal lobe epilepsy," Human Pathology, vol. 32, no. 6, pp. 643-648, 2001.

[5] J. Maher and R. S. McLachlan, "Febrile convulsions: is seizure duration the most important predictor of temporal lobe epilepsy?” Brain, vol. 118, no. 6, pp. 1521-1528, 1995.

[6] H.-G. Wieser, "Mesial temporal lobe epilepsy with hippocampal sclerosis," Epilepsia, vol. 45, no. 6, pp. 695-714, 2004.

[7] M. Thom, "Neuropathological findings in epilepsy," Current Diagnostic Pathology, vol. 10, no. 2, pp. 93-105, 2004.

[8] T. Eid, A. Williamson, T. S. W. Lee, O. A. Petroff, and N. C. De Lanerolle, "Glutamate and astrocytes-key players in human mesial temporal lobe epilepsy?" Epilepsia, vol. 49, no. 2, pp. $42-52,2008$. 
[9] A. R. Wyler, F. C. Dohan, J. B. Schweitzer, and A. D. Berry, "A grading system for mesial temporal pathology (hippocampal sclerosis) from anterior emporal lobectomy," Journal of Epilepsy, vol. 5, no. 4, pp. 220-225, 1992.

[10] I. Blümcke, E. Pauli, H. Clusmann et al., "A new clinicopathological classification system for mesial temporal sclerosis," Acta Neuropathologica, vol. 113, no. 3, pp. 235-244, 2007.

[11] M. Thom, S. Eriksson, L. Martinian et al., "Temporal lobe sclerosis associated with hippocampal sclerosis in temporal lobe epilepsy: neuropathological features," Journal of Neuropathology and Experimental Neurology, vol. 68, no. 8, pp. 928-938, 2009.

[12] J. H. Margerison and J. A. N. Corsellis, "Epilepsy and the temporal lobes: a clinical, electroencephalographic and neuropathological study of the brain in epilepsy, with particular reference to the temporal lobes," Brain, vol. 89, no. 3, pp. 499$530,1966$.

[13] L. P. Hudson, D. G. Munoz, L. Miller, R. S. McLachlan, J. P. Girvin, and W. T. Blume, "Amygdaloid sclerosis in temporal lobe epilepsy," Annals of Neurology, vol. 33, no. 6, pp. 622-631, 1993.

[14] J. Engel Jr., W. J. Brown, and D. E. Kuhl, "Pathological findings underlying focal temporal lobe hypometabolism in partial epilepsy," Annals of Neurology, vol. 12, no. 6, pp. 518-528, 1982.

[15] D. M. Yilmazer-Hanke, H. K. Wolf, J. Schramm, C. E. Elger, O. D. Wiestler, and I. Blümcke, "Subregional pathology of the amygdala complex and entorhinal region in surgical specimens from patients with pharmacoresistant temporal lobe epilepsy," Journal of Neuropathology and Experimental Neurology, vol. 59, no. 10, pp. 907-920, 2000.

[16] M. F. Levesque, N. Nakasato, H. V. Vinters, and T. L. Babb, "Surgical treatment of limbic epilepsy associated with extrahippocampal lesions: the problem of dual pathology," Journal of Neurosurgery, vol. 75, no. 3, pp. 364-370, 1991.

[17] A. A. Raymond, D. R. Fish, J. M. Stevens, M. J. Cook, S. M. Sisodiya, and S. D. Shorvon, "Association of hippocampal sclerosis with cortical dysgenesis in patients with epilepsy," Neurology, vol. 44, no. 10, pp. 1841-1845, 1994.

[18] F. Cendes, M. J. Cook, C. Watson et al., "Frequency and characteristics of dual pathology in patients with lesional epilepsy," Neurology, vol. 45, no. 11, pp. 2058-2064, 1995.

[19] L. M. Li, F. Cendes, F. Andermann et al., "Surgical outcome in patients with epilepsy and dual pathology," Brain, vol. 122, no. 5, pp. 799-805, 1999.

[20] S. M. Mirsattari, D. A. Steven, J. Keith, and R. R. Hammond, "Pathophysiological implications of focal cortical dysplasia of end folium for hippocampal sclerosis," Epilepsy Research, vol. 84, no. 2-3, pp. 268-272, 2009.

[21] I. Blümcke, H. V. Vinters, D. Armstrong, E. Aronica, M. Thom, and R. Spreafico, "Malformations of cortical development and epilepsies: neuropathological findings with emphasis on focal cortical dysplasia," Epileptic Disorders, vol. 11, no. 3, pp. 181193, 2009.

[22] A. J. Barkovich, R. I. Kuzniecky, G. D. Jackson, R. Guerrini, and W. B. Dobyns, "A developmental and genetic classification for malformations of cortical development," Neurology, vol. 65, no. 12, pp. 1873-1887, 2005.

[23] Y. J. Jiang, L.-C. Ang, and W. T. Blume, "Extent of EEG epileptiform pattern distribution in "focal" cortical dysplasia," Journal of Clinical Neurophysiology, vol. 27, no. 5, pp. 309-311, 2010.

[24] D. H. Lee, F. Q. Gao, J. M. Rogers et al., "MR in temporal lobe epilepsy: analysis with pathologic confirmation," American Journal of Neuroradiology, vol. 19, no. 1, pp. 19-27, 1998.
[25] B. Pasquier, M. Péoch'h, B. Fabre-Bocquentin et al., "Surgical pathology of drug-resistant partial epilepsy: a 10-yearexperience with a series of 327 consecutive resections," Epileptic Disorders, vol. 4, no. 2, pp. 99-119, 2002.

[26] V. Salanova, O. Markand, and R. Worth, "Temporal lobe epilepsy: analysis of patients with dual pathology," Acta Neurologica Scandinavica, vol. 109, no. 2, pp. 126-131, 2004.

[27] Y. S. Piao, D. H. Lu, L. Chen et al., "Neuropathological findings in intractable epilepsy: 435 Chinese cases," Brain Pathology, vol. 20, no. 5, pp. 902-908, 2010.

[28] R. A. Prayson, R. Spreafico, and H. V. Vinters, "Pathologic characteristics of the cortical dysplasias," Neurosurgery Clinics of North America, vol. 13, no. 1, pp. 17-25, 2002.

[29] R. S. Fisher and D. Blum, "Epilepsy surgery where there is dual pathology," Lancet, vol. 354, no. 9175, pp. 267-268, 1999.

[30] I. Blümcke and A. Mühlebner, "Neuropathological work-up of focal cortical dysplasias using the new ILAE consensus classification system - practical guideline article invited by the Euro-CNS Research committee," Clinical Neuropathology, vol. 30, no. 4, pp. 164-177, 2011.

[31] A. Palmini, I. Najm, G. Avanzini et al., "Terminology and classification of the cortical dysplasias," Neurology, vol. 62, no. 6, pp. S2-S8, 2004.

[32] W. A. Chamberlain, M. L. Cohen, K. A. Gyure et al., "Interobserver and intraobserver reproducibility in focal cortical dysplasia (malformations of cortical development)," Epilepsia, vol. 50, no. 12, pp. 2593-2598, 2009.

[33] S. L. Ding, G. W. Van Hoesen, M. D. Cassell, and A. Poremba, "Parcellation of human temporal polar cortex: a combined analysis of multiple cytoarchitectonic, chemoarchitectonic, and pathological markers," Journal of Comparative Neurology, vol. 514, no. 6, pp. 595-623, 2009.

[34] I. Blümcke, M. Thom, E. Aronica et al., "The clinicopathologic spectrum of focal cortical dysplasias: a consensus classification proposed by an ad hoc Task Force of the ILAE Diagnostic Methods Commission," Epilepsia, vol. 52, no. 1, pp. 158-174, 2011.

[35] S. Sisodiya, "Epilepsy: the new order-classifying focal cortical dysplasias," Nature Reviews Neurology, vol. 7, no. 3, pp. 129130, 2011.

[36] L. Tassi, B. Pasquier, L. Minotti et al., "Cortical dysplasia: electroclinical, imaging, and neuropathologic study of 13 patients," Epilepsia, vol. 42, no. 9, pp. 1112-1123, 2001.

[37] F. Al Sufiani, Y. Jiang, W. T. Blume, and L. Ang, "Institutional review of epilepsy resection specimens with focal cortical dysplasia," The Canadian Journal of Neurological Sciences, vol. 39, no. 1, p. 106, 2012.

[38] M. Thom, S. Sisodiya, W. Harkness, and F. Scaravilli, "Microdysgenesis in temporal lobe epilepsy: a quantitative and immunohistochemical study of white matter neurones," Brain, vol. 124, no. 11, pp. 2299-2309, 2001.

[39] S. H. Eriksson, K. Malmgren, and C. Nordborg, "Microdysgenesis in epilepsy," Acta Neurologica Scandinavica, vol. 111, no. 5, pp. 279-290, 2005.

[40] A. M. Rojiani, J. A. Emery, K. J. Anderson, and J. K. Massey, "Distribution of heterotopic neurons in normal hemispheric white matter: a morphometric analysis," Journal of Neuropathology and Experimental Neurology, vol. 55, no. 2, pp. 178183, 1996.

[41] J. A. Emery, S. N. Roper, and A. M. Rojiani, "White matter neuronal heterotopia in temporal lobe epilepsy: a morphometric and immunohistochemical study," Journal of Neuropathology and Experimental Neurology, vol. 56, no. 12, pp. 1276-1282, 1997. 
[42] B. S. Kasper, H. Stefan, M. Buchfelder, and W. Paulus, "Temporal lobe microdysgenesis in epilepsy versus control brains," Journal of Neuropathology and Experimental Neurology, vol. 58, no. 1, pp. 22-28, 1999.

[43] R. Spreafico and I. Blümcke, "Focal Cortical Dysplasias: clinical implication of neuropathological classification systems," Acta Neuropathologica, vol. 120, no. 3, pp. 359-367, 2010.

[44] S. Wiebe, D. G. Munoz, S. Smith, and L. Donald H, "Meningioangiomatosis. A comprehensive analysis of clinical and laboratory features," Brain, vol. 122, no. 4, pp. 709-726, 1999.

[45] R. A. Prayson, "Meningioangiomatosis: a clinicopathologic study including MIB1 immunoreactivity," Archives of Pathology and Laboratory Medicine, vol. 119, no. 11, pp. 1061-1064, 1995.

[46] A. Perry, B. Kurtkaya-Yapicier, B. W. Scheithauer et al., "Insights into meningioangiomatosis with without meningioma: a clinicopathologic end genetic series of 24 cases with review of the literature," Brain Pathology, vol. 15, no. 1, pp. 55-65, 2005.

[47] W. A. Hauser and J. P. Mohr, "Editorial: seizures, epilepsy, and vascular malformations," Neurology, vol. 76, no. 18, pp. 15401541, 2011.

[48] K. Upchurch, J. M. Stern, N. Salamon et al., "Epileptogenic temporal cavernous malformations: operative strategies and postoperative seizure outcomes," Seizure, vol. 19, no. 2, pp. 120-128, 2010.

[49] E. E. Volk and R. A. Prayson, "Hamartomas in the setting of chronic epilepsy: a clinicopathologic study of 13 cases," Human Pathology, vol. 28, no. 2, pp. 227-232, 1997.

[50] I. Blümcke, M. Löbach, H. K. Wolf, and O. D. Wiestler, "Evidence for developmental precursor lesions in epilepsyassociated glioneuronal tumors," Microscopy Research and Technique, vol. 46, no. 1, pp. 53-58, 1999.

[51] A.-C. Yang, K. Zhang, J.-G. Zhang et al., "Temporal lobe epilepsy with hypothalamic hamartoma: a rare case," Chinese Medical Journal, vol. 124, no. 7, pp. 1114-1117, 2011.

[52] H. K. Wolf, M. G. Campos, J. Zentner et al., "Surgical pathology of temporal lobe epilepsy. Experience with 216 cases," Journal of Neuropathology and Experimental Neurology, vol. 52, no. 5, pp. 499-506, 1993.

[53] S. Uliel-Sibony, U. Kramer, I. Fried, A. Fattal-Valevski, and S. Constantini, "Pediatric temporal low-grade glial tumors: epilepsy outcome following resection in 48 children," Child's Nervous System, vol. 27, no. 9, pp. 1413-1418, 2011.

[54] A. Furuta, H. Takahashi, F. Ikuta, K. Onda, N. Takeda, and R. Tanaka, "Temporal lobe tumor demonstrating ganglioglioma and pleomorphic xanthoastrocytoma components. Case report," Journal of Neurosurgery, vol. 77, no. 1, pp. 143 147, 1992.

[55] Y. Shimbo, H. Takahashi, M. Hayano, T. Kumagai, and S. Kameyama, "Temporal lobe lesion demonstrating features of dysembryoplastic neuroepithelial tumor and ganglioglioma: a transitional form?" Clinical Neuropathology, vol. 16, no. 2, pp. 65-68, 1997.

[56] R. A. Prayson, J. Fong, and I. Najm, "Coexistent pathology in chronic epilepsy patients with neoplasms," Modern Pathology, vol. 23, no. 8, pp. 1097-1103, 2010.

[57] U. P. Kalyan-Raman and W. C. Olivero, "Ganglioglioma: a correlative clinicopathological and radiological study of ten surgically treated cases with follow-up," Neurosurgery, vol. 20, no. 3, pp. 428-433, 1987.

[58] I. Blümcke, K. Giencke, E. Wardelmann et al., "The CD34 epitope is expressed in neoplastic and malformative lesions associated with chronic, focal epilepsies," Acta Neuropathologica, vol. 97, no. 5, pp. 481-490, 1999.

[59] I. Blümcke and O. D. Wiestler, "Gangliogliomas: an intriguing tumor entity associated with focal epilepsies," Journal of Neuropathology and Experimental Neurology, vol. 61, no. 7, pp. 575-584, 2002.

[60] R. Demarchi, S. Abu-Abed, D. Munoz, and R. Loch MacDonald, "Malignant ganglioglioma: case report and review of literature," Journal of Neuro-Oncology, vol. 101, no. 2, pp. 311318, 2011.

[61] M. Thom, A. Toma, S. An et al., "One hundred and one dysembryoplastic neuroepithelial tumors: an adult epilepsy series with immunohistochemical, molecular genetic, and clinical correlations and a review of the literature," Journal of Neuropathology and Experimental Neurology, vol. 70, no. 10, pp. 859-878, 2011.

[62] R. A. Prayson and M. L. Estes, "Dysembryoplastic neuroepithelial tumor," American Journal of Clinical Pathology, vol. 97, no. 3, pp. 398-401, 1992.

[63] A. R. Campos, H. Clusmann, M. Von Lehe et al., "Simple and complex dysembryoplastic neuroepithelial tumors (DNT) variants: clinical profile, MRI, and histopathology," Neuroradiology, vol. 51, no. 7, pp. 433-443, 2009.

[64] T. N. Chandrashekhar, A. Mahadevan, S. Vani et al., "Pathological spectrum of neuronal/glioneuronal tumors from a tertiary referral neurological Institute," Neuropathology, vol. 32, no. 1, pp. 1-12, 2012.

[65] R. R. Hammond, N. Duggal, J. M. J. Woulfe, and J. P. Girvin, "Malignant transformation of a dysembryoplastic neuroepithelial tumor: case report," Journal of Neurosurgery, vol. 92, no. 4, pp. 722-725, 2000.

[66] E. J. Rushing, L. D. Thompson, and H. Mena, "Malignant transformation of a dysembryoplastic neuroepithelial tumor after radiation and chemotherapy," Annals of Diagnostic Pathology, vol. 7, no. 4, pp. 240-244, 2003.

[67] R. A. Prayson, "Papillary glioneuronal tumor," Archives of Pathology and Laboratory Medicine, vol. 124, no. 12, pp. 1820$1823,2000$.

[68] J. Pimentel, C. Barroso, J. Miguens, C. Firmo, and J. L. Antunes, "Papillary glioneuronal tumor-prognostic value of the extension of surgical resection," Clinical Neuropathology, vol. 28, no. 4, pp. 287-294, 2009.

[69] J. J. Kepes, L. J. Rubinstein, and L. F. Eng, "Pleomorphic xanthoastrocytoma: a distinctive meningocerebral glioma of young subjects with relatively favorable prognosis. A study of 12 cases," Cancer, vol. 44, no. 5, pp. 1839-1852, 1979.

[70] C. Giannini, B. W. Scheithauer, M. B. S. Lopes, T. Hirose, J. M. Kros, and S. R. VandenBerg, "Immunophenotype of pleomorphic xanthoastrocytoma," American Journal of Surgical Pathology, vol. 26, no. 4, pp. 479-485, 2002.

[71] D. J. Wallace, R. W. Byrne, D. Ruban, E. J. Cochran, D. Roh, and W. W. Whisler, "Temporal lobe pleomorphic xanthoastrocytoma and chronic epilepsy: long-term surgical outcomes," Clinical Neurology and Neurosurgery, vol. 113, no. 10, pp. 918922, 2011.

[72] C. Bayindir, N. Balak, A. Karasu, and D. Kasaroglu, "Anaplastic pleomorphic xanthoastrocytoma," Child's Nervous System, vol. 13 , no. 1 , pp. $50-56,1997$.

[73] R. A. Prayson and H. H. Morris, "Anaplastic pleomorphic xanthoastrocytoma," Archives of Pathology and Laboratory Medicine, vol. 122, no. 12, pp. 1082-1086, 1998.

[74] G. A. Alexiou, M. Moschovi, K. Stefanaki, C. Prodromou, G. Sfakianos, and N. Prodromou, "Malignant progression of 
a pleomorphic xanthoastrocytoma in a child," Neuropediatrics, vol. 41 , no. 2 , pp. 69-71, 2010.

[75] H. Martinez-Diaz, B. K. Kleinschmidt-DeMasters, S. Z. Powell, and A. T. Yachnis, "Giant cell glioblastoma and pleomorphic xanthoastrocytoma show different immunohistochemical profiles for neuronal antigens and p53 but share reactivity for class III $\beta$-tubulin," Archives of Pathology and Laboratory Medicine, vol. 127, no. 9, pp. 1187-1191, 2003.

[76] M. Wang, T. Tihan, A. M. Rojiani et al., "Monomorphous angiocentric glioma: a distinctive epileptogenic neoplasm with features of infiltrating astrocytoma and ependymoma," Journal of Neuropathology and Experimental Neurology, vol. 64, no. 10 , pp. 875-881, 2005.

[77] M. Preusser, A. Hoischen, K. Novak et al., "Angiocentric glioma: report of clinico-pathologic and genetic findings in 8 cases," American Journal of Surgical Pathology, vol. 31, no. 11, pp. 1709-1718, 2007.

[78] J. Schramm, C. Luyken, H. Urbach, R. Fimmers, and I. Blümcke, "Evidence for a clinically distinct new subtype of grade II astrocytomas in patients with long-term epilepsy," Neurosurgery, vol. 55, no. 2, pp. 340-347, 2004.

[79] I. Blümcke, C. Luyken, H. Urbach, J. Schramm, and O. D. Wiestler, "An isomorphic subtype of long-term epilepsy-associated astrocytomas associated with benign prognosis," Acta Neuropathologica, vol. 107, no. 5, pp. 381-388, 2004.

[80] J. Englander, T. Bushnik, T. T. Duong et al., "Analyzing risk factors for late posttraumatic seizures: a prospective, multicenter investigation," Archives of Physical Medicine and Rehabilitation, vol. 84, no. 3, pp. 365-373, 2003.

[81] R. D'Ambrosio and E. Perucca, "Epilepsy after head injury," Current Opinion in Neurology, vol. 17, no. 6, pp. 731-735, 2004.

[82] H. Payan, M. Toga, and M. Bérard-Badier, "The pathology of post-traumatic epilepsies,” Epilepsia, vol. 11, no. 1, pp. 81-94, 1970.

[83] R. P. Lesser, H. Luders, D. S. Dinner, and H. H. Morris, "Epileptic seizures due to thrombotic and embolic cerebrovascular disease in older patients," Epilepsia, vol. 26, no. 6, pp. 622-630, 1985.

[84] C. F. Bladin and J. W. Norris, "Stroke, seizures/epilepsy," in Primer on Cerebrovascular Diseases, K. M. A. Welch, L. R. Caplan, D. J. Reis, B. K. Siesjo, and W. Bryce, Eds., pp. 355360, Academic Press, San Diego, Calif, USA, 1997.

[85] M. A. Farrell, O. Droogan, D. Lenard Secor, V. Poukens, B. Quinn, and H. V. Vinters, "Chronic encephalitis associated with epilepsy: immunohistochemical and ultrastructural studies," Acta Neuropathologica, vol. 89, no. 4, pp. 313-321, 1995.

[86] S. Duishanbai, D. Jiafu, H. Guo et al., "Intracranial hydatid cyst in children: report of 30 cases," Child's Nervous System, vol. 26, no. 6, pp. 821-827, 2010.

[87] M. M. Bianchin, T. R. Velasco, L. Wichert-Ana, O. M. Takayanagui, J. P. Leite, and A. C. Sakamoto, "How frequent is the association of neurocysticercosis and mesial temporal lobe epilepsy with hippocampal sclerosis?" Epilepsia, vol. 51, no. 11, pp. 2359-2360, 2010.

[88] A. Vezzani and T. Granata, "Brain inflammation in epilepsy: experimental and clinical evidence," Epilepsia, vol. 46, no. 11, pp. 1724-1743, 2005.

[89] E. Aronica and P. B. Crino, "Inflammation in epilepsy: clinical observations," Epilepsia, vol. 52, supplement 3, pp. 26-32, 2011.

[90] R. A. Prayson and J. L. Frater, "Rasmussen encephalitis: a clinicopathologic and immunohistochemical study of seven patients," American Journal of Clinical Pathology, vol. 117, no. 5, pp. 776-782, 2002.

[91] C. A. Pardo, E. P. G. Vining, L. Guo, R. L. Skolasky, B. S. Carson, and J. M. Freeman, "The Pathology of Rasmussen Syndrome: stages of cortical involvement and neuropathological studies in 45 hemispherectomies," Epilepsia, vol. 45, no. 5, pp. 516-526, 2004.

[92] C. G. Bien, J. Bauer, T. L. Deckwerth et al., "Destruction of neurons by cytotoxic T cells: a new pathogenic mechanism in Rasmussen's encephalitis," Annals of Neurology, vol. 51, no. 3, pp. 311-318, 2002. 


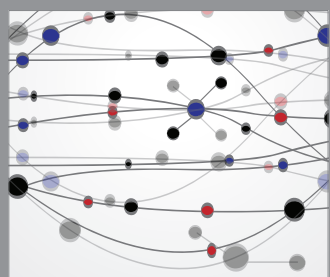

The Scientific World Journal
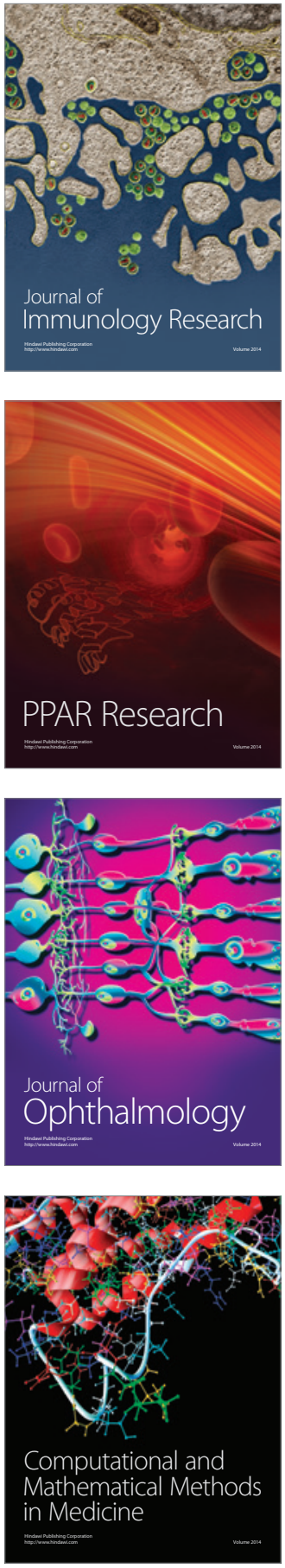

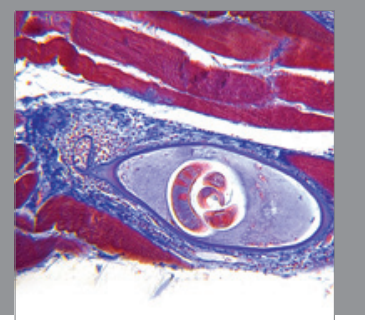

Gastroenterology

Research and Practice
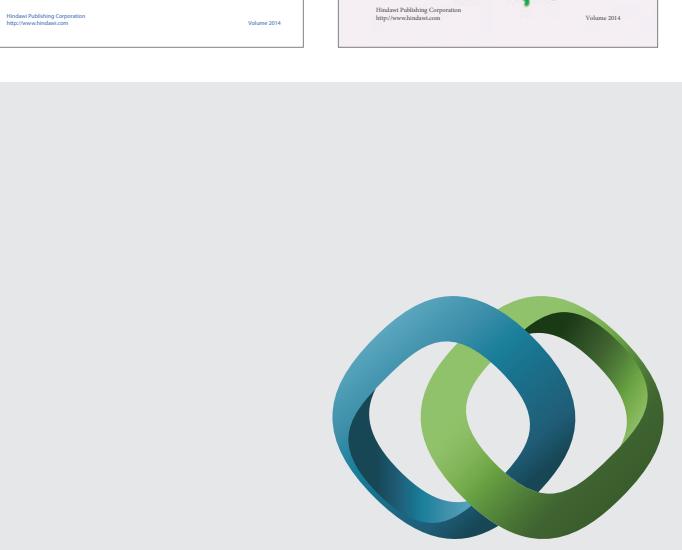

\section{Hindawi}

Submit your manuscripts at

http://www.hindawi.com
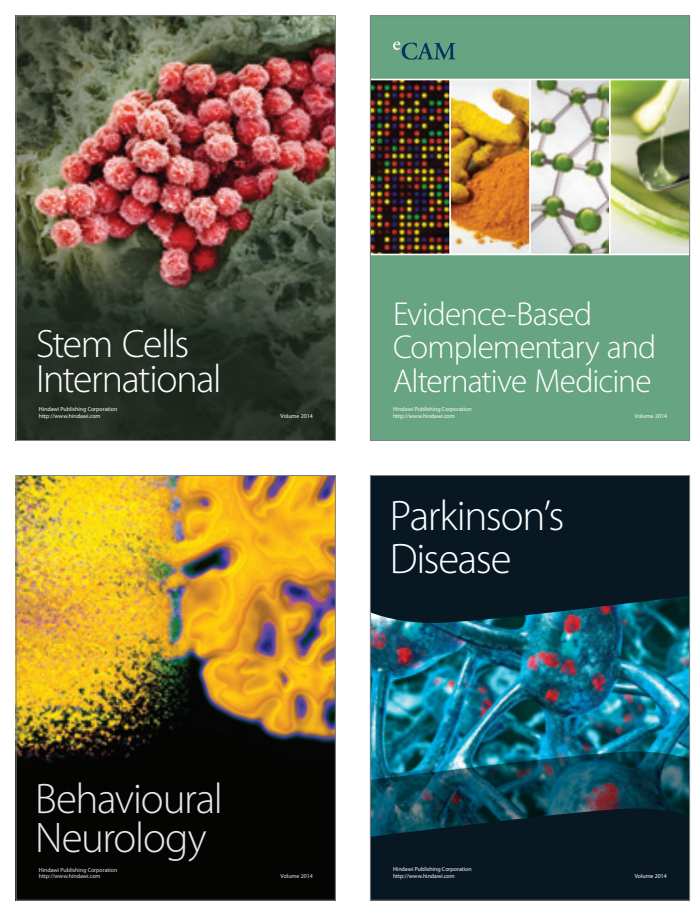

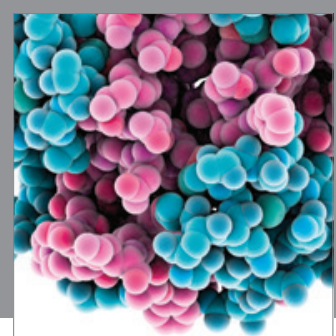

Journal of
Diabetes Research

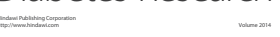

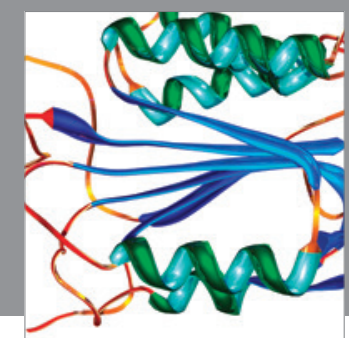

Disease Markers
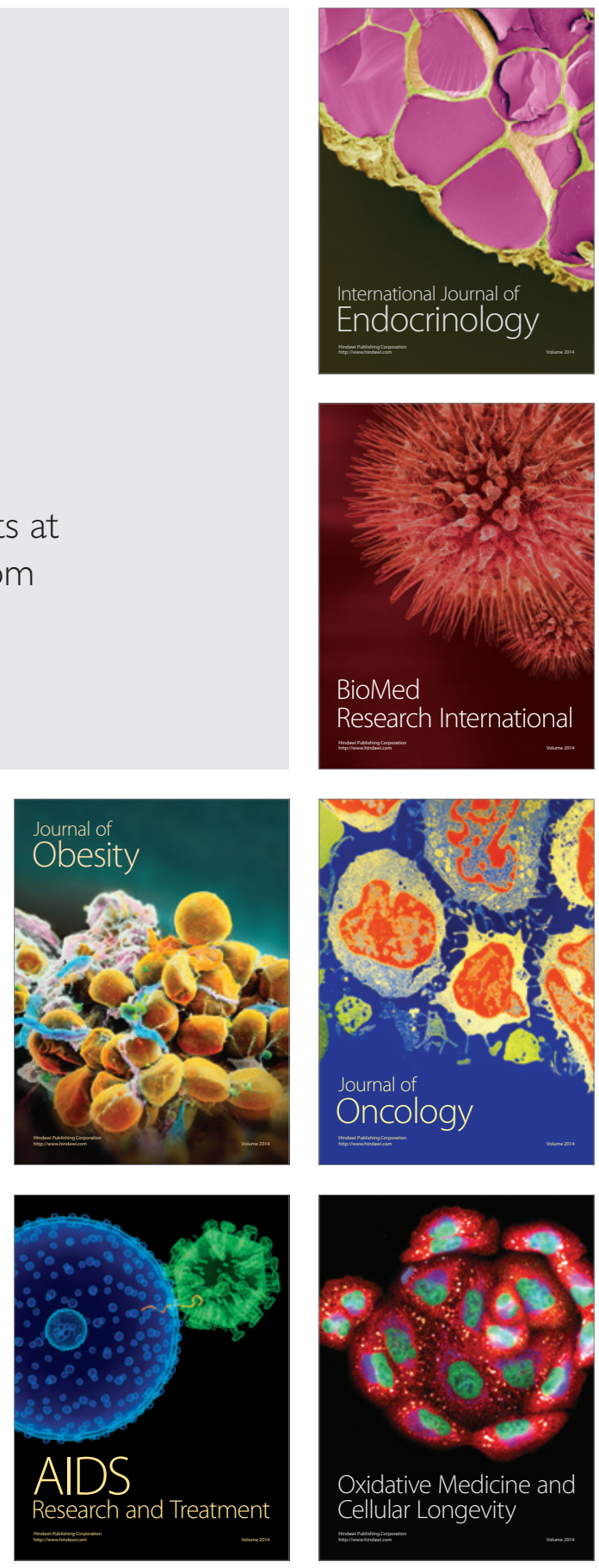